\title{
Comparison of different pellet injection systems for ELM pacing
}

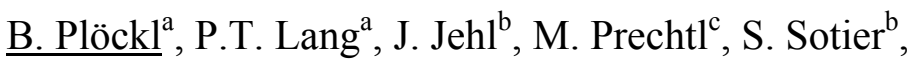 \\ ASDEX Upgrade team ${ }^{\mathrm{a}}$ and JET EFDA Contributors* \\ a Max Planck-Institut für Plasmaphysik, EURATOM Association, Boltzmannstraße 2, 85748 Garching, Germany \\ b Hochschule München, University of Applied Sciences - München, Lothstr. 34, 80335 München, Germany \\ c Hochschule Coburg, Fakultät Maschinenbau, Friedrich-Streib-Strasse 2, 96450 Coburg, Germany \\ Corresponding author: bernhard.ploeckl@ipp.mpg.de
}

ELM control in ITER is important to avoid the rapid erosion of plasma facing components (PFC) and core plasma pollution. Pacing and mitigation of ELMs can be achieved by injection of cryogenic deuterium pellets into the plasma. Successful control relies on precisely timed reliable delivery of pellets with reasonable size and velocity. To minimize unwanted fuelling constraints high speed and small pellet size seem of advantage. Efforts to develop pellet launcher systems optimised for pacing purposes have been undertaken, based on the two major accelerator types, the centrifuge and the gas or blower gun.

\section{Introduction}

Control or avoidance of Edge Localized Modes (ELMs) is one of the most important research tasks in view of ITER to avoid the rapid erosion of plasma facing components (PFC) and core plasma pollution [1]. In order to mitigate ELM related power fluxes below the hazard level for divertor lifetime, the strategy is either to avoid ELMs at all or to gain control over the ELM size by enhancing their frequency accordingly. One control method considered in the ITER baseline is pellet injection. It was found that the injection of cryogenic ice pellets from D fuel can reliably trigger ELMs; ELM frequency control and size reduction was demonstrated in several tokamaks $[2 ; 3]$. Nonetheless, the pellet ELM pacing tool needs further optimisation since the required high repetition rates combined with large pellet sizes (investigated pellet systems previously used for fuelling) can cause unwanted side effects. The major concern is pellet particle fuelling changing plasma parameters unfavourable in the edge and the enhanced particle throughput expending large fractions of pump and Tritium recovery capacities.

Looking at pellet pacing as a control tool, it is obvious that all plasma parameters and the requested pellet repetition rate have to be considered as given boundary conditions. Remaining parameters disposable for tool optimisation are pellet mass $\left(\mathrm{m}_{\mathrm{P}}\right)$, speed $\left(\mathrm{v}_{\mathrm{P}}\right)$ and the injection location. Further on, quality of the control tool depends on the delivery reliability (arriving pellets per request), mass losses in the transfer guide system and $\mathrm{v}_{\mathrm{P}}$ variation. Missing pellets or others arriving with little mass will result in a temporary loss of ELM control while $v_{P}$ variations cause an irregular pellet train and hence ELM pattern. Consequently, the consideration of a pellet ELM pacing system should cover the injector capabilities but also its interplay with the transfer system and the resulting plasma response.

At present the premise of a technically robust, well matured and reliable system as required for ITER is only met by two major injector types: gas (blower) guns and mechanical accelerators relying usually on centrifugal force acceleration. Both types are under investigation at the IPP with focus on their application for pellet ELM pacing. This paper reports our latest results, discusses the status and perspectives for both approaches and finally an outlook on our optimization strategy.

\section{Gas Guns}

\subsection{AUG blower gun: working principle and results}

In case of a blower gun (and a gas gun as well), acceleration of pellets is due to expanding gas which pushes or drags the pellet. The diameter of the pellet is smaller than the diameter of the barrel and the gas flows around the pellet. Acceleration force transfer follows the force closure principle (frictional connection), hence some slip is present. The thermal impact of the propellant gas influences the pellet as well. Both effects can cause a variation of the velocity and time scatter.

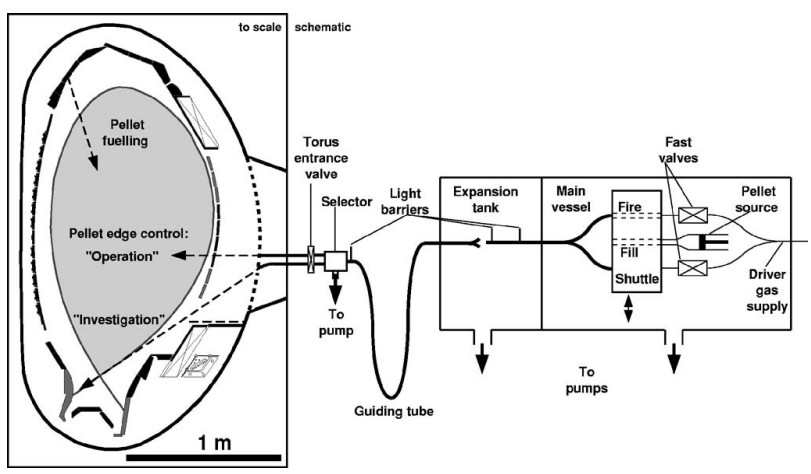

Fig. 1. Overview of the experimental setup at ASDEX Upgrade. Left part (to scale): Poloidal cross section of AUG in the 2006 configuration with a typical plasma configuration foreseen for ELM investigation experiments. Right (schematic only): Blower gun injector delivering pellets to the torus entrance port.

A shuttle in reciprocating motion cuts the ice rod and moves it in one of the two firing positions in front of the barrel. A short propellant gas pulse released by a fast

Author's email: bernhard.ploeckl@ipp.mpg.de

* See the Appendix of F. Romanelli et al., Proceedings of the $22^{\text {nd }}$ IAEA FEC 2008, Geneva, Switzerland 
valve accelerates the pellet. Best results were achieved by operating the shuttle at a temperature of about $85 \mathrm{~K}$. Although this temperature is far beyond the sublimation temperature of a $\mathrm{D}_{2}$ pellet (about $18 \mathrm{~K}$ in the given pressure range) [4], the Leidenfrost effect prevents fast ablation. The pellet source is composed of two cryostat systems, one for the extrusion of an (cylindrical) ice rod and one for storing this ice rod. Both cryostats are cooled by liquid Helium down to a temperature of about $5-10 \mathrm{~K}$. Triggered by a pellet request, the extruded ice rod in the storage cryostat is pushed out by a mechanical lever towards the shuttle for filling.

The pellet system originally designed for fueling size was modified to get smaller pellet sizes for ELM mitigation experiments. Therefore the pellet diameter was reduced from $2 \mathrm{~mm}$ diameter $\left(\sim 1.6 \times 10^{20} \mathrm{D}\right)$ down to $1 \mathrm{~mm}$ diameter $\left(\sim 0.2 \times 10^{20} \mathrm{D}\right)$. The pellet velocity range spans from $100 \mathrm{~m} / \mathrm{s}$ up to $350 \mathrm{~m} / \mathrm{s}$. The maximal repetition rate is $143 \mathrm{~Hz}$ ( $7 \mathrm{~ms}$ cycle time).

The transfer system guides the pellets to the low field side (LFS) of the AUG vacuum vessel. The guiding system consists of a Teflon tube with $6 \mathrm{~mm}$ inner diameter supported by and pumped through stainless steel tubes. The length of transfer system is about $5 \mathrm{~m}$. Video diagnostic access and light barriers for time of flight measurements are installed at the entrance ("D1") and the end ("D2") of the transfer system. Using these tools, the velocity, velocity scatter and efficiency of pellet trains can be measured. Arrival of the pellet at the plasma is detected by a pellet ablation monitor.

It turned out, that the pellet efficiency decreases strongly with decreasing pellet size. In case of small pellets only about $50 \%$ of the requested pellets leave the transfer line at D2. In the torus tube, further $50 \%$ of the pellets are damaged. The resulting yield (pellets at plasma) hence is only $25 \%$ of the requested pellets.

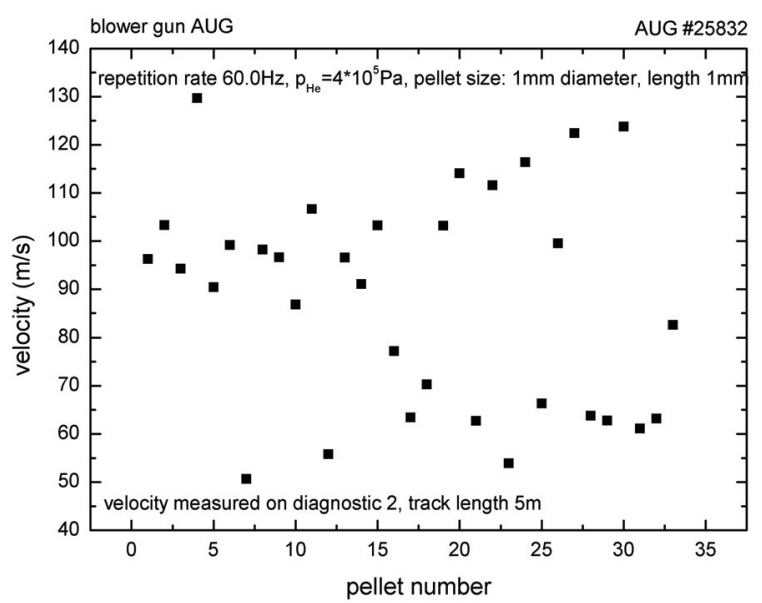

Fig. 2: Pellet velocity distribution of a pellet train arriving at D2.

Typical pellet velocities achieved for a fixed propellant gas pressure of $4 \cdot 10^{5} \mathrm{~Pa}$ (valve opening time $1 \mathrm{~ms}$ ) at $60 \mathrm{~Hz}$ repetition rate were found to scatter from $50 \mathrm{~m} / \mathrm{s}$ up to $130 \mathrm{~m} / \mathrm{s}$. The resulting time-of-flight in the transfer tube (length $5 \mathrm{~m}$ ) is hence $38.5 \mathrm{~ms}$ for the fastest and up to $100 \mathrm{~ms}$ for the slowest pellets. The flight time scatter within the tube is thus $61.5 \mathrm{~ms}$. At a repetition rate of $60 \mathrm{~Hz}$, the nominal temporal distance between two pellets is $16 \mathrm{~ms}$, much less than the time scatter.

\subsection{JET HFPI: system and preliminary results}

The new JET HFPI pellet launcher [5] follows the same basic principle as described for the AUG blower gun. Up to now, the maximum achieved repetition rate was $20 \mathrm{~Hz}$, the maximum pellet velocity $300 \mathrm{~m} / \mathrm{s}$.

The transfer system consists of 3 tracks: low field side (LFS, $10 \mathrm{~m}$ length), vertical high field side (VHFS, $20 \mathrm{~m}$ length) and high field side (HFS) [5]. The latter is actually inhibited for safety reasons.

Analyzing a good ELM pacing experiment performed using fuelling size LFS pellets at a repetition rate of $10 \mathrm{~Hz}$ and an average pellet velocity of $142 \mathrm{~m} / \mathrm{s}$, we found the time scatter of pellets arriving on plasma was $\pm 9 \mathrm{~ms}( \pm 2 \sigma$, i.e. 2 standard deviations $)$.

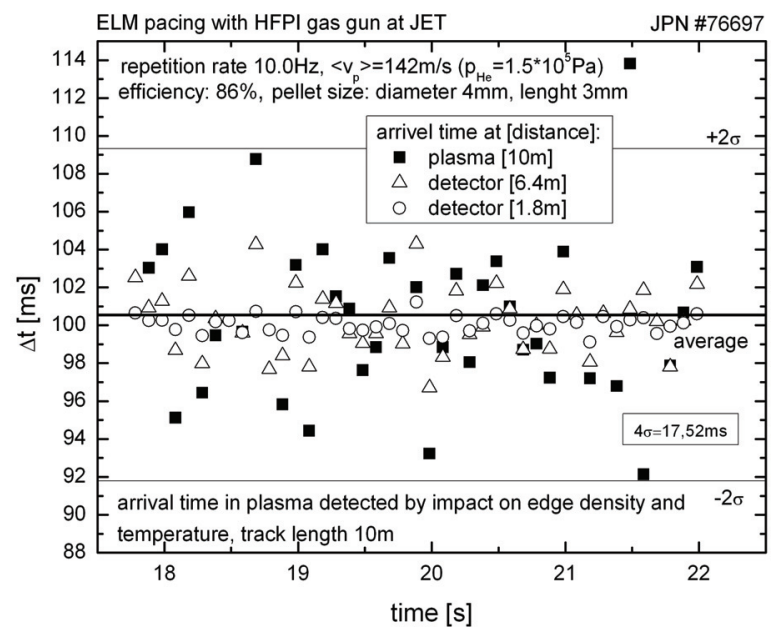

Figure 3: ELM pacing at JET using outboard launch fuelling size pellets from the new gas gun type HFPI system. The observed time scatter on plasma is $\pm 8.76 \mathrm{~ms}$ $( \pm 2 \sigma)$. The increasing time scatter during the pellet flight can be seen as well from two passed detectors.

\subsection{Discussion of the AUG Blower Gun}

For pellet sizes envisaged for ELM pacing in current tokamaks, the thermal energy content of the propellant gas pulse can be much higher than the energy required for the entire pellet sublimation. A fraction of this energy is transferred to the pellet while a flow of propellant gas is applied for the acceleration. This can cause already significant pellet ablation and as a consequence change in the pellet mass and increasing slip.

In addition, ice production and storage units are thermally coupled to the acceleration unit (tic-tac- like moving shuttle). The shuttle temperature has a large influence on the gun performance. The optimum temperature is found to be around $85 \mathrm{~K}$. The thermal balance of both acceleration channels is very important to achieve narrow velocity distribution. 


\section{Centrifuge}

\subsection{Working principle}

In the centrifuge the acceleration is due to the centrifugal force acting on a pellet sliding in the groove of a rotating straight arm. Consequently there is no slip during acceleration. Hence the acceleration of pellets follows the form-closure principle (positive locking) and can be very precisely tuned provided the onset conditions are sufficiently accurate. This requirement is attained by the stop cylinder technique [6]. The generated pellet drops into the stop cylinder that is mounted adjustable but not rotating above the centrifuge. This stop cylinder ensures an accurate timing for the start of the acceleration since the pellet leaves the stop cylinder at a well defined radial position with zero speed. In the absence of any propellant gas and due to the Leidenfrost effect heat transfer and friction are low. The centrifuge revolution frequency precisely prescribes the pellet velocity (speed scatter at the exit $<0.25 \%$ ); the repetition rate has to be an integer fraction of centrifuge frequency.

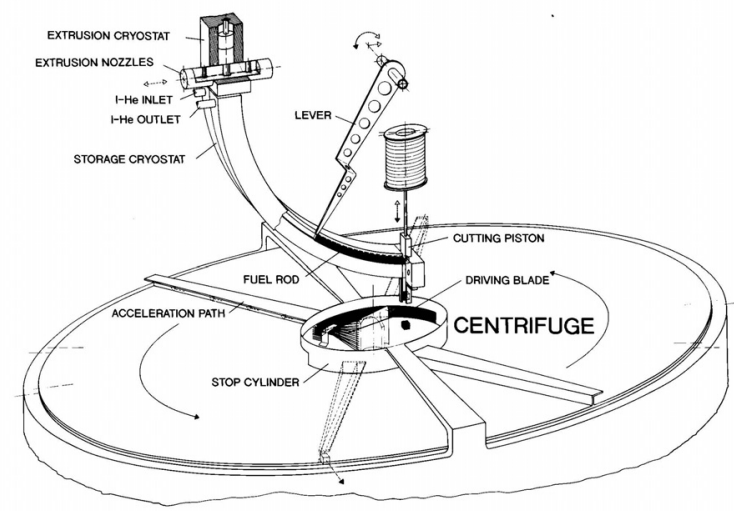

Fig. 4: Extrusion cryostat, movable 3 way nozzle, lever and cutter. The stop cylinder mounted above the rotating arm is thermally disconnected from both cryostats.

Upon request, the ice rod is shifted by a stepping motor driven mechanical lever; the number of steps determining the pellet length. The pellet is chopped from the rod using an electromechanical cutter.

The ice production is similar to the blower gun using an extrusion cryostat and a storage cryostat. Three different pellet sizes can be selected without any mechanical intervention into the system [7].

The transfer line guides the pellets to the high field side (HFS) of AUG. In order to achieve high pellet velocities a "looping" has been installed, a $17 \mathrm{~m}$ long transfer line of elliptical shape avoiding changing curvature direction. Due to the Leidenfrost effect and good pumping, friction and the heat transfer are low. The maximum useful transfer velocity is $1000 \mathrm{~m} / \mathrm{s}$ [8].

\subsection{AUG centrifuge: system and results}

The centrifuge originally designed as fueling system could be adapted for pacing needs due to the large flexibility of the system. It is capable to produce cubical pellets with side length of $1.4 ; 1.65$ and $1.9 \mathrm{~mm}$ corresponding to nominal pellet particle contents of 1.6; 2.7 and $4.1 \times 10^{20} \mathrm{D}$ atoms. The accessible pellet velocity range spans from $240 \mathrm{~m} / \mathrm{s}$ to $1200 \mathrm{~m} / \mathrm{s}$. In the actual configuration, there are four preselected velocity values available: $240,600,880$ and $1000 \mathrm{~m} / \mathrm{s}$; an ongoing modification foresees arbitrary speed choice. An upper speed limit of $1000 \mathrm{~m} / \mathrm{s}$ is set by the looping. Furthermore, resonances in the mechanical system would cause strong wearing for operation in the range 650 to $800 \mathrm{~m} / \mathrm{s}$ and some other small frequency band. Hence, operation will be envisaged only within some distinct frequency ranges.

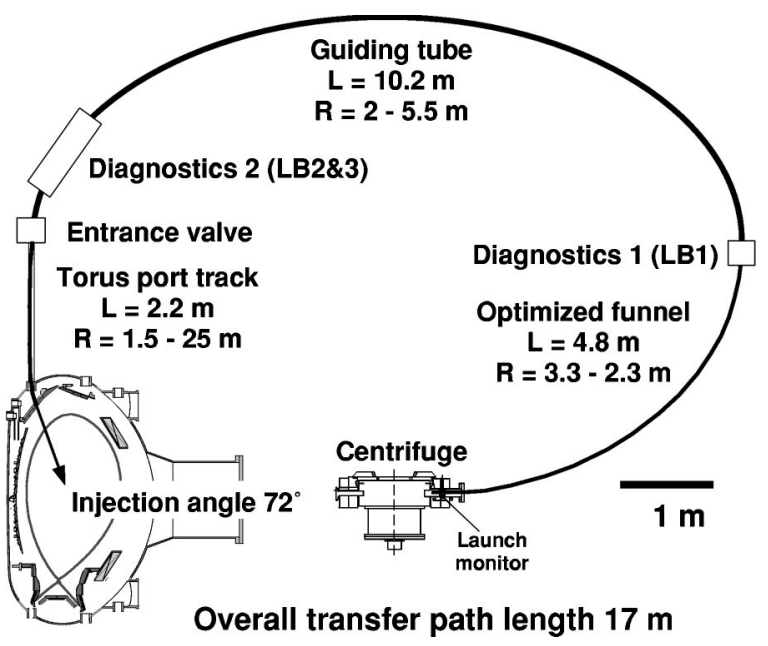

Fig. 5: "Looping" transfer line, actual configuration on AUG for injection of pellets from the high field side (HFS)

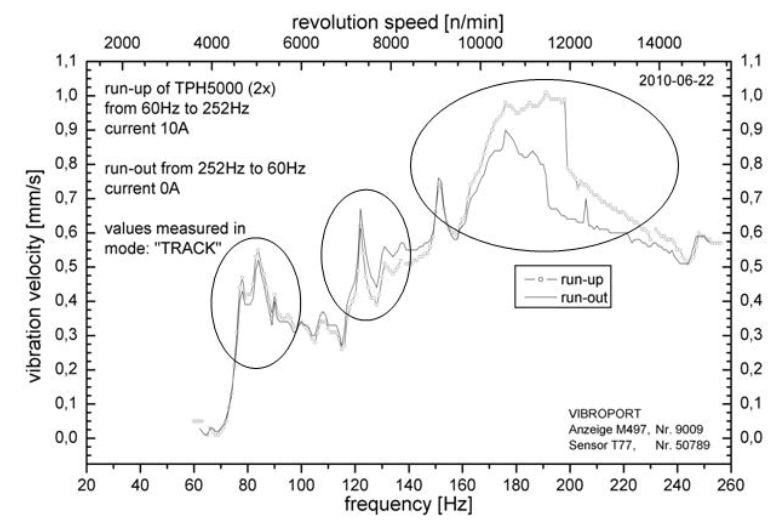

Figure 6: Vibration measurements on AUG centrifuge, peak wearing bands to be avoided for operation are indicated.

The maximum possible repetition rate is $250 \mathrm{~Hz}$, i.e. launching one pellet per centrifuge revolution. Also, there is a limitation due to excessive heating of the cutter coil at high frequencies. For this reason, the highest repetition rate used is $\sim 80 \mathrm{~Hz}$.

The centrifuge delivery efficiency was found to be beyond 0.9 for every launch velocity. For velocities up to $880 \mathrm{~m} / \mathrm{s}$ the overall transfer efficiency is typically larger than 0.8 , above it can decrease to 0.6. For the selected example shown in Fig. 7 (AUG\#17455), careful optimization achieved a value of 0.98 at $997 \mathrm{~m} / \mathrm{s}$ for $62 \mathrm{~Hz}$ pellet rate. The time scatter of pellet arrival into 
plasma is $\pm 0.23 \mathrm{~ms}( \pm 2 \sigma)$. Thus, with this system, pacing experiments applying high repetition rates up to several hundred $\mathrm{Hz}$ would be possible.

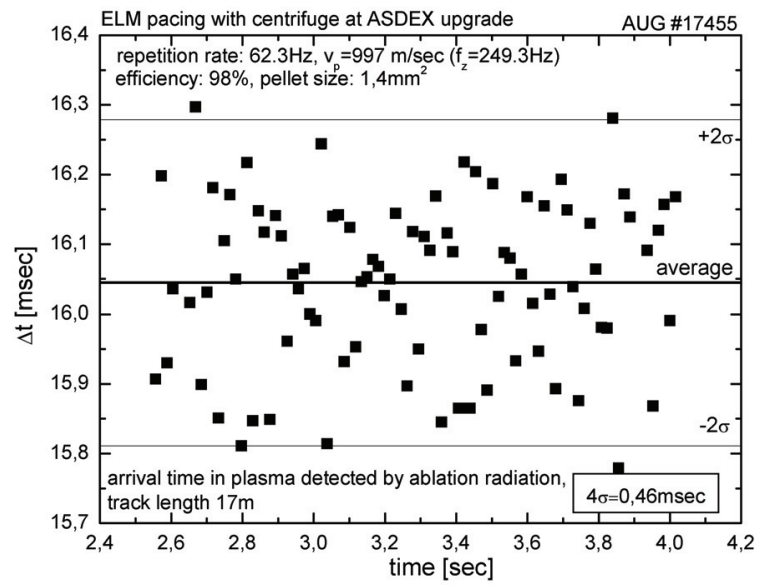

Fig. 7: ELM pacing at AUG using inboard launch from the centrifuge system. The observed time scatter on plasma is $\pm 0.22 \mathrm{~ms}( \pm 2 \sigma)$. Total pellet flight time is $17 \mathrm{~ms}$. With the speed scatter observed in this system pacing rates up to several hundred $\mathrm{Hz}$ are potentially accessible.

\subsection{JET centrifuge: system and results}

The JET centrifuge is an upgraded version of the AUG centrifuge, where the ice was produced and delivered from a long pulse extruder [9].

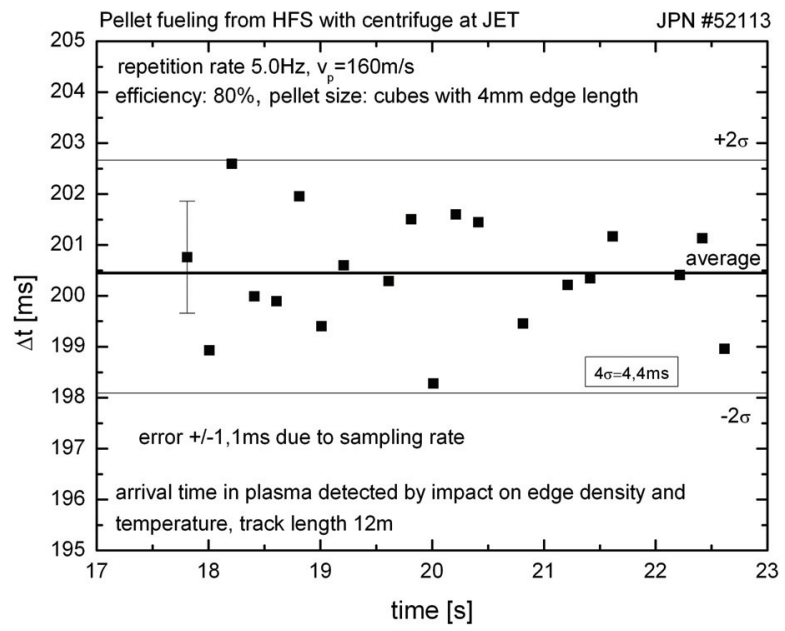

Fig. 8: Pellet fueling at JET using HFS launch from the centrifuge system. The observed time scatter on plasma is $\pm 2.2 \mathrm{~ms}( \pm 2 \sigma)$, about equal to the temporal resolution achieved for the arrival detection. Hence, the real time scatter is probably even lower.

\subsection{Discussion}

The pellet acceleration in the centrifuge is very precise due to the form closure principle (positive locking). Since there is no propellant gas and different parts are thermally separated, the ice is kept well insulated inside the storage cryostat. The Leidenfrost effect keeps the heat transfer low during acceleration and transfer. Thus the pellet mass variation is low - and in combination with the precise launch velocity ensures the velocity scatter to stay small. Using a movable nozzle providing 3 different extrusion cross sections, the pellet diameter can be changed without any intervention into the mechanical system. The pellet length can be adjusted simply by varying the number of stepping motor steps.

\section{Conclusion}

For ELM pacing using pellet injection, precise timing of pellets is crucial. The initial time precision of the pellet acceleration source, i.e. blower gun or centrifuge, is deteriorated along the transfer path. The worsening of the time precision constrains the construction design and the length of the transfer line.

Thorough investigations at AUG were done to characterize the two injection principles complemented by the corresponding JET systems. Table 1 presents the summary on the time scatter characteristics of these systems at AUG and JET.

\begin{tabular}{|c|c|c|c|c|}
\hline System & $\begin{array}{c}\text { Pellet } \\
\text { velocity } \\
{[\mathrm{m} / \mathrm{s}]}\end{array}$ & $\begin{array}{c}\text { repetition } \\
\text { rate } \\
{[\mathrm{Hz}]}\end{array}$ & $\begin{array}{c}\text { Transfer: } \\
\text { length } / \\
\text { time }\end{array}$ & $\begin{array}{c}\text { Time } \\
\text { scatter } \\
{[\mathrm{ms}]}\end{array}$ \\
\hline $\begin{array}{c}\text { AUG } \\
\text { centrifuge }\end{array}$ & 1000 & 62 & $\begin{array}{c}17 \mathrm{~m} \\
17 \mathrm{~ms}\end{array}$ & \pm 0.23 \\
\hline $\begin{array}{c}\text { AUG } \\
\text { blower gun }\end{array}$ & 100 & 60 & $\begin{array}{c}5 \mathrm{~m} \\
50 \mathrm{~ms}\end{array}$ & \pm 31 \\
\hline $\begin{array}{c}\text { JET } \\
\text { centrifuge }\end{array}$ & 160 & 5 & $\begin{array}{c}12 \mathrm{~m} \\
75 \mathrm{~ms}\end{array}$ & \pm 2.2 \\
\hline $\begin{array}{c}\text { JET } \\
\text { gas gun }\end{array}$ & 142 & 10 & $\begin{array}{c}10 \mathrm{~m} \\
70 \mathrm{~ms}\end{array}$ & \pm 9 \\
\hline
\end{tabular}

Table 1: Comparison of relevant data for the AUG and JET pellet injection systems.

The pellet injection system based on the centrifuge configuration - at both experiments - has significantly lower time scatter and thus higher maximum pellet frequency, primarily related to the acceleration principle. Although not discussed here, the geometry of the transfer system has to be considered as well, as it can significantly influence the time scatter.

In the future, based on the presented results the AUG experiment will enforce fuelling and ELM pacing investigation using the centrifuge system. The JET HFPI gas gun is designed for pacing experiments with an elevated repetition rate coming as close as possible to the planned 50-60Hz.

\section{References}

[1] N. Klimov et al., J. Nucl. Mater. 390-391, 2009, 721

[2] P.T. Lang et al., Nucl. Fusion 44 (2004) 665

[3] L.R. Baylor et al., Nucl. Fusion 47 (2007) 1598

[4] P. T. Lang et al., Rev. of Sci. Instruments, vol. 78, (2007), pp 023504

[5] A. Geraud et.al., Fusion Engineering and Design 82 (2007) 2183-2188

[6] C. Andelfinger et al., Rev. Sci. Instruments, vol. 64 (1993) 983-989

[7] P.T. Lang and P. Cierpka, Rev. Sci. Instruments., vol 67, (1996) 619-620

[8] P. T. Lang et.al., Rev. of Sci. Instruments, vol 74 (2003) 3977-3983

[9] D.J. Wilson et al., 20th IEEE/NPSS SOFE San Diego (2003) p 86. 